\title{
Étude du cycle biologique d'un parasite fongique des poissons
}

\author{
par G. CHAUVIER \\ Sous-Directeur de la Ménagerie du Muséum national d'Histoire naturelle, \\ 57, rue Cuvier, 75005 Paris \\ (Collaboration technique : $\mathrm{M}^{\mathrm{me}} \mathrm{J}$. Mortier-Gabet)
}

RESUME. Le parasite fongique isolé des poissons présente, en culture pure, une particularité remarquable, la coexistence de trois modalités de reproduction sexuée. Il peut, en effet :

a. effectuer une caryogamie dans des éléments dicaryons qui sont, soit de petits éléments levuriformes, soit de courts tubes mycéliens issus d'éléments levuriformes plus volumineux ;

b. conjuguer deux isogamètes levuriformes arrondis et aboutir à la formation d'un ookyste où se forment des endospores ;

c. fusionner deux hyphes issues de deux gamètes circulaires et produire, après cette somatogamie, un sporange où se forment des endospores.

\section{Study of the life cycle of a fongic parasite from fishes.}

SUMMARY. In pure culture the parasitic fungus isolated from fish shows a remarkable peculiarity for it can: a) exhibit caryogamy in dicaryotic elements that are either small yeast-like cells or short mycelial tubes expanding from larger yeasts; b) conjugate two yeastlike circular isogametes and produce thereafter an oocyst in which endospores take form; c) merge two hyphae expanding from two round cells and produce by means of this somatogamous process a sporangium in which endospores take form.

Dans une note préliminaire $\left(^{*}\right)$, nous avons signalé l'existence d'une mycose viscérale mortelle de poissons dulçaquicoles tropicaux. Le microorganisme responsable a pu être entretenu en culture depuis trois ans sur milieu glucosé de Sabouraud et sur quelques milieux liquides et nous avons pu suivre presque jour après jour son évolution. Outre les formes déjà décrites, observées en début de culture (plasmodes, élé-

Accepté le 16 mars 1980.

(*) Ann. Parasitol. Hum. Comp., 1979, 54 (1), 105-111. 
A

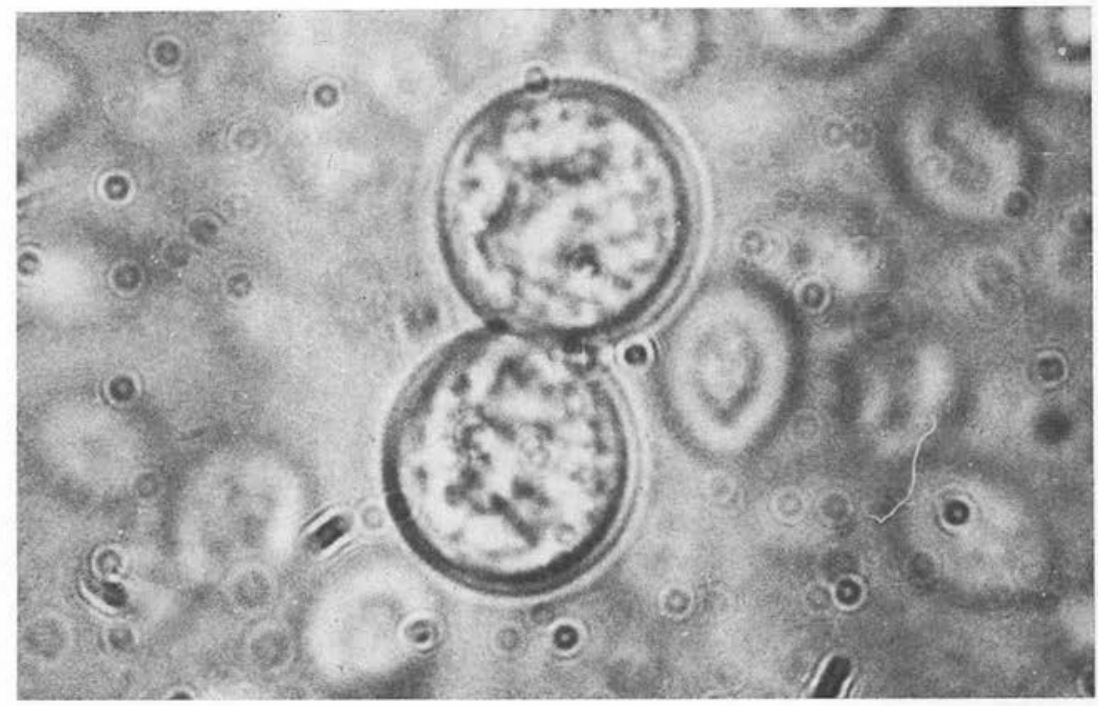

B

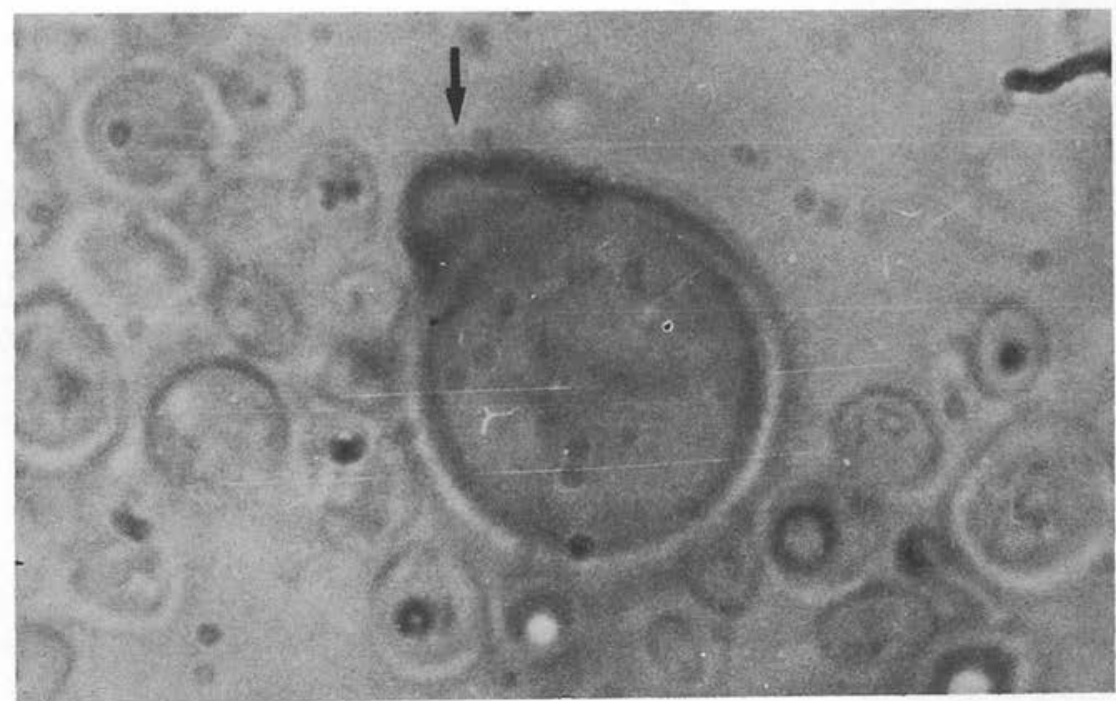

Fig. 1. A : Conjugaison de 2 gamètes (début de fusion); B : Ichthyosporidium (Ichthyophonus) sp. Ookyste jeune montrant le reliquat de membrane de l'un des gamètes. 
ments levuriformes, ébauches mycéliennes, formes de résistance), nous avons pu mettre en évidence l'existence d'une série de formes correspondant très nettement à des manifestations de sexualité. En effet, à maintes reprises nous avons pu observer les phénomènes suivants :

1. Accolement et fusion progressive de deux éléments levuriformes circulaires, aboutissant à la formation d'un ookyste, entouré d'une membrane relativement épaisse, et caractérisé par la présence d'une sorte "d'anse de théière " qui n'est, en fait, que le reliquat de la membrane de l'un des gamètes (schéma 1 ; fig. $1 \mathrm{~A}$ et $1 \mathrm{~B}$ ). Quelques stades de la fusion des gamètes ont pu être observés (fig. $2 A, B, C, D, E$ ). Les deux

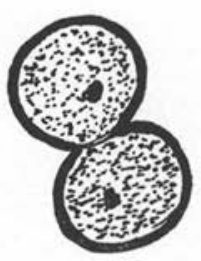

A

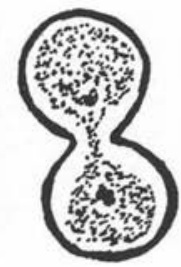

B

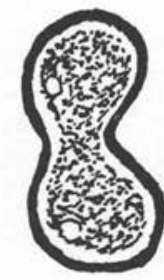

C

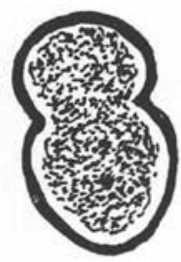

D

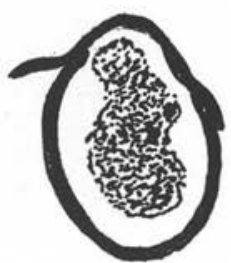

$\mathrm{E}$

Fig. 2. - Stades de fusion des gamètes.

A : accolement des gamètes ; B : début de fusion cytoplasmique, après lyse des membranes ; C, D : pénétration progressive du contenu d'une cellule dans l'autre ; E : stade final, la membrane du gamète supérieur forme une sorte de couvercle sur l'ookyste.

gamètes étant de taille et de structure apparemment identiques, il s'agit donc d'un phénomène de gamétogamie, avec isogamie, des plus classiques ( $f$ ig. 3 A et $3 \mathrm{~B}$ ).

2. Germination de deux éléments levuriformes circulaires, avec formation de deux hyphes tubulaires qui se rencontrent, fusionnent, donnent ensuite naissance à un filament unique, non cloisonné, qui ne tarde pas à se renfler en massue (schéma 2) ou en ampoule (schéma 3). Dans ces renflements sont situées de petites masses cytoplasmiques pourvues d'un gros noyau que nous assimilons aux "endospores mycéliennes" (hyphal bodies) décrites par d'autres auteurs $(1,4)$. Ces endospores sont destinées à être libérées dans le milieu extérieur, sous forme de "sphérules " contenant de petits plasmodes, qui s'en échappent à leur tour, après rupture de la membrane, pour mener une vie libre.

Ces processus de fusion d'hyphes aboutissant à la formation d'un sporange peut être défini par le terme de "Somatogamie». Ici aussi une phase haploïde est représentée par les deux éléments gamétiques filamenteux, la phase diploïde par le sporange et les spores.

3. A partir d'éléments levuriformes ovoïdes à membrane épaisse (chlamydospores), bourgeonnement d'éléments levuriformes plus petits, à membrane mince, et pourvus chacun de deux noyaux (schéma 4). 
A

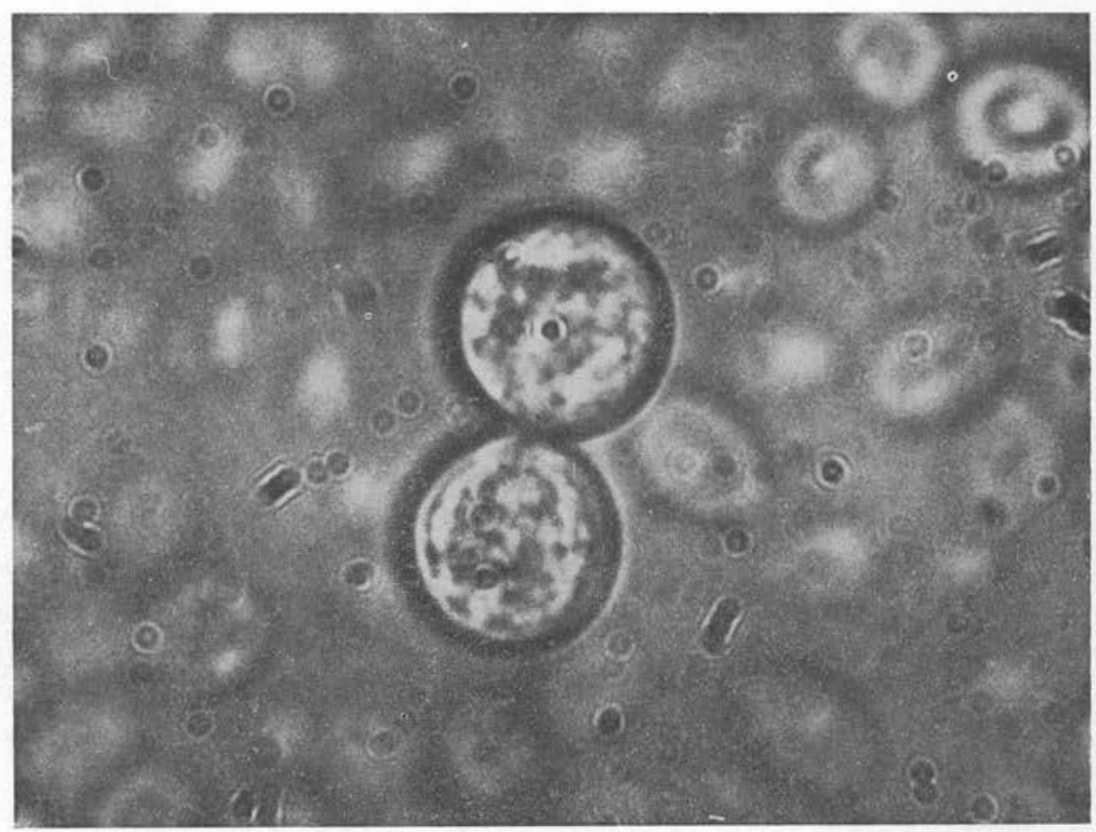

B

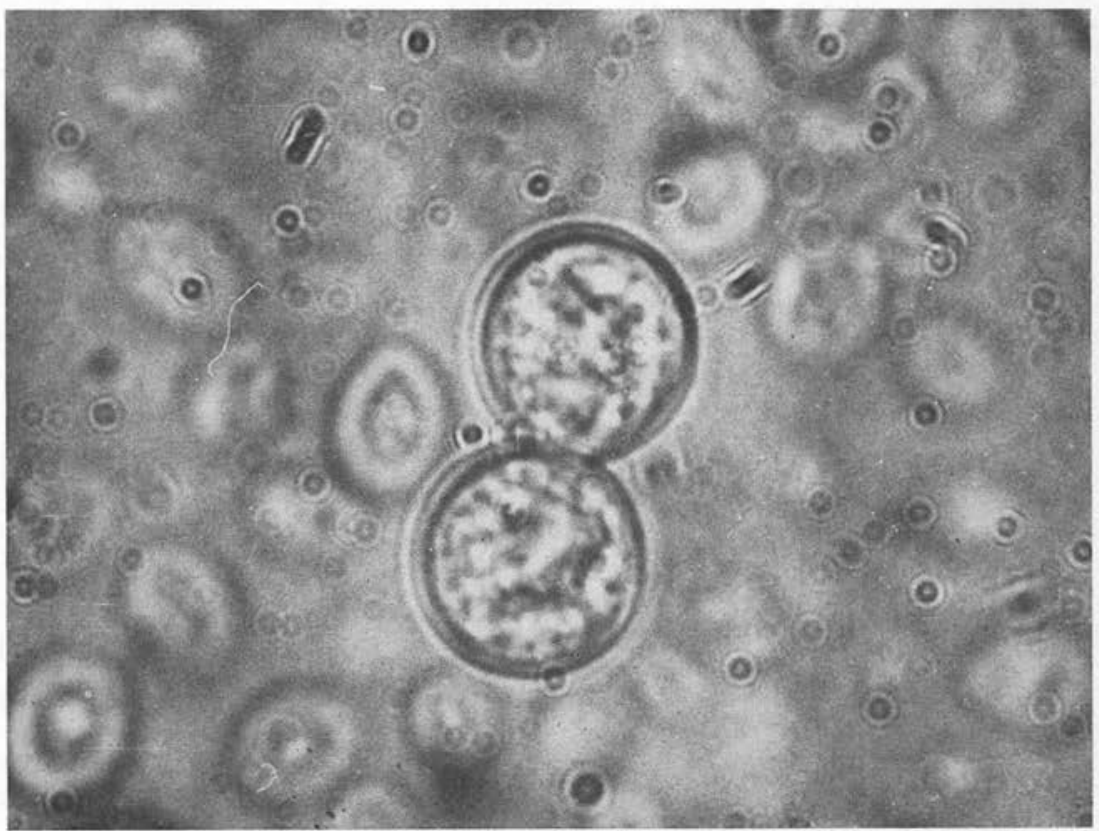

Fig. 3. A : Conjugaison de gamètes (accolement); B : Conjugaison de 2 gamètes (début de fusion). 


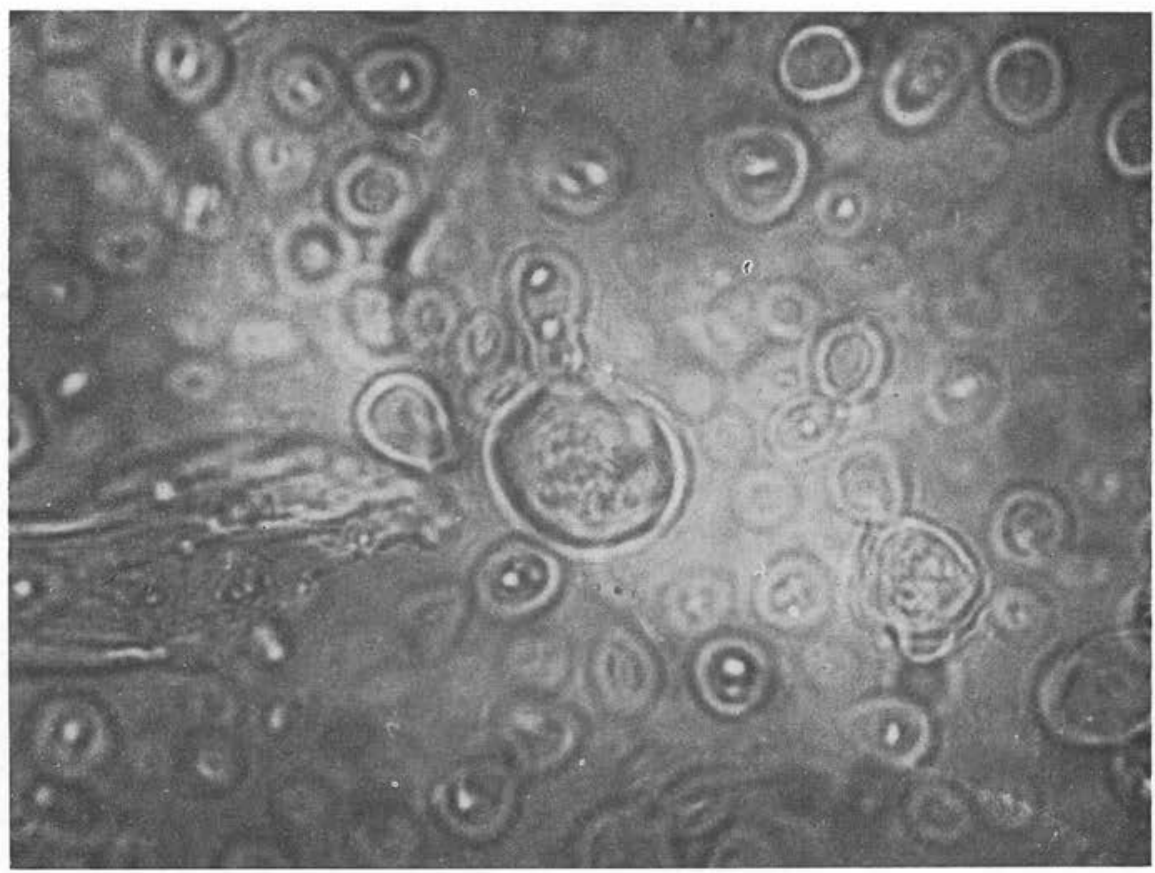

Fig. 4. - Ichthyophonus sp. Chlamydospore avec germination tubulaire renfermant un dicaryon.

4. Germinations tubulaires issues d'éléments levuriformes circulaires et contenant un cytoplasme granuleux ainsi que deux noyaux, proches de l'extrémité du filament (schéma 5 ; fig. 4).

5. Germinations tubulaires issues d'éléments levuriformes circulaires et contenant de nombreuses endospores (schéma 6).

6. Condensations cytoplasmiques multiples, pourvues chacune d'un noyau, à l'intérieur d'un gros élément levuriforme circulaire (schéma 7 ; fig. 5).

7. Fusion des noyaux (caryogamie) dans les éléments levuriformes binucléés issus, soit du bourgeonnement de chlamydospores (cf. $g 3$ et schéma 4), soit d'une condensation cytoplasmique dans un plasmode (schéma 8). Evolution ultérieure de ces éléments levuriformes de deux façons différentes:

a. par fragmentation du noyau unique ainsi créé, vers la formation d'un petit plasmode plurinucléé inclus dans une «sphérule ";

$b$. par fragmentation du noyau unique, accompagnée de condensations cytoplasmiques, aboutissant à la formation d'endospores, signalées au g 6 (schéma 7 ; fig. 5).

On peut admettre, croyons-nous, que les aspects signalés aux $g 4^{\circ}$ et $5^{\circ}$ (schémas 5 et 6) représentent le stade initial et le stade final d'un processus de caryo- 


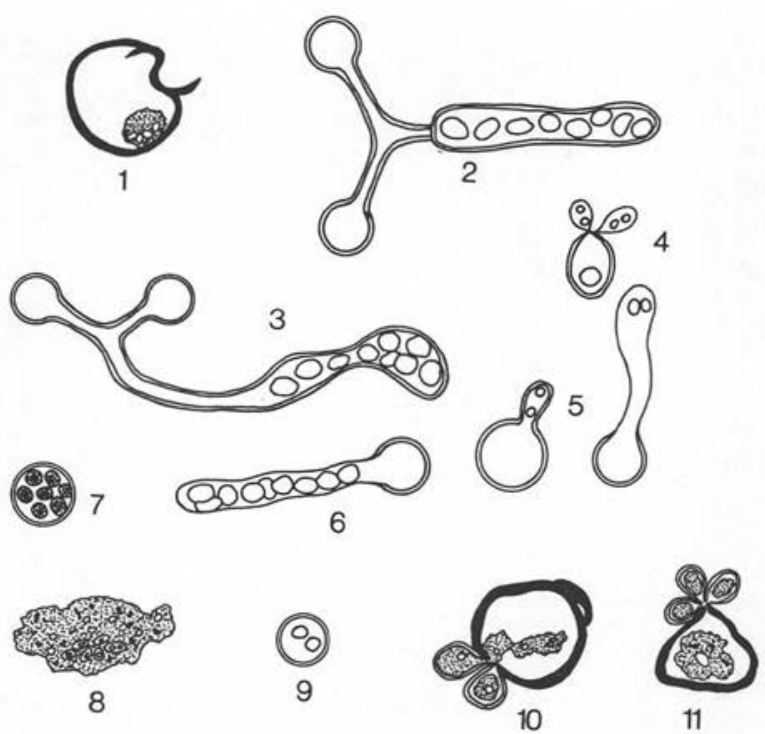

Schémas 1 à 11. - Formes observées in vitro, liées aux trois modes de reproduction sexuée. 1 , ookyste après gamétogamie ; 2,3 , sporanges avec endospores, après somatogamie ; 4,5 , formes dicaryons ; 6 , sporange, après caryogamie ; 7 , microsporange $; 8$, dicaryon inclus dans un plasmode; 9, dicaryon libre; 10, émission de spores par un ookyste; 11, émission de gamètes par un sporokyste.

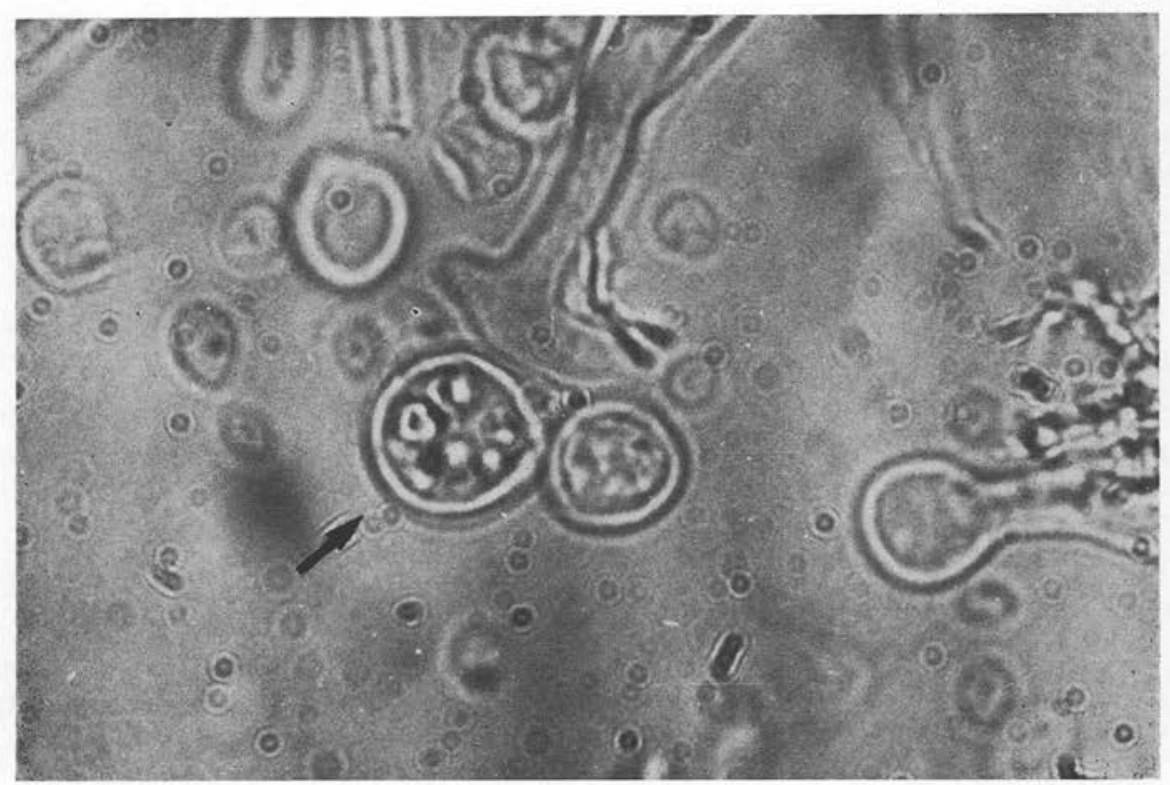

Fig. 5. - Endospores dans un élément levuriforme circulaire. 
gamie survenant dans un filament et aboutissant à la formation d'endospores mycéliennes. Il n'est pas impossible qu'un élément levuriforme circulaire, très nettement binucléé (comme celui que représente le schéma 9) puisse évoluer, selon les conditions du milieu, tantôt en émettant un tube germinatif où se produira la caryogamie, tantôt en réalisant cette caryogamie sans germination préalable, d'où la réalisation d'un "micro-sporange " contenant un petit nombre d'endospores (cf. $g$ et fig. 5). Il peut paraître surprenant qu'une même espèce fongique puisse présenter à la fois trois modes de reproduction sexuée : gamétogamie, somatogamie et caryogamie. Nous avons nous-mêmes longtemps hésité avant d'admettre ce fait, mais la répétition d'observations le confirmant nous y contraint. Nous sommes donc amenés à proposer l'explication suivante du cycle du parasite :

\section{I. - Phase diploïde.}

a. Forme végétative libre : le plasmode, capable de produire des éléments conidiens;

b. Eléments conidiens, ovoïdes, uni-nucléés, parfois binucléés (stade dicaryon);

c. Eléments levuriformes circulaires résultant du développement, après caryogamie, des conidies binucléées;

d. Eléments amiboïdes de petite taille issus des éléments conidiens uninucléés ;

$e$. Ebauches mycéliennes en forme de quilles, capables de produire des bourgeons uninucléés ;

f. Ookystes résultant de la gamétogamie ou de la somatogamie et renfermant les endospores ;

g. Endospores issues des ookystes et capables de donner issue à de petits plasmodes, ou d'évoluer en chlamydospores ;

$h$. Chlamydospores ou Sporokystes, résultant de l'évolution d'endospores dans un milieu devenu défavorable à la culture, et pouvant donner des gamètes.

\section{II. - Phase haploïde.}

Eléments levuriformes circulaires capables de fusionner deux à deux, soit directement (gamétogamie), soit après formation d'un tube germinatif (somatogamie).

Ces éléments sont nécessairement le résultat d'une méïose ; celle-ci doit survenir dans les sporokystes avant l'émission par ceux-ci d'éléments levuriformes arrondis (schéma 11).

D'autre part, il est nécessaire d'admettre qu'une méïose se produit aussi de temps à autre dans les plasmodes d'où la production par ceux-ci de quelques éléments conidiens bi-nucléés (haplo-diploïdes ?) ou bien dans les chlamydospores, d'où le bourgeonnement de formes levures bi-nucléées.

Ce cycle biologique ayant ainsi été défini par culture in vitro, il importe maintenant de chercher à voir comment le parasite l'accomplit dans les conditions naturelles. L'infection d'un poisson sain doit pouvoir se produire de diverses façons (fig. 6). Divers auteurs [Fish (1934), Euzéby (1969), etc.] ont pensé que le mode de contamination 
le plus probable doit être l'ingestion de la chair et des viscères d'un autre poisson, ou d'un petit crustacé, lui-même parasité. Ceci paraît très vraisemblable et conduit à admettre la séquence suivante d'événements: dissolution des membranes enveloppant les kystes parasitaires, sous l'action des sucs digestifs ; libération des plasmodes qu'elles contenaient : production d'éléments conidiens par les plasmodes; libération de petites formes amiboïdes par les conidies uninucléées; pénétration de ces formes amiboïdes dans la circulation générale du poisson, d'où leur dissémination dans tout l'organisme, y compris le système nerveux.

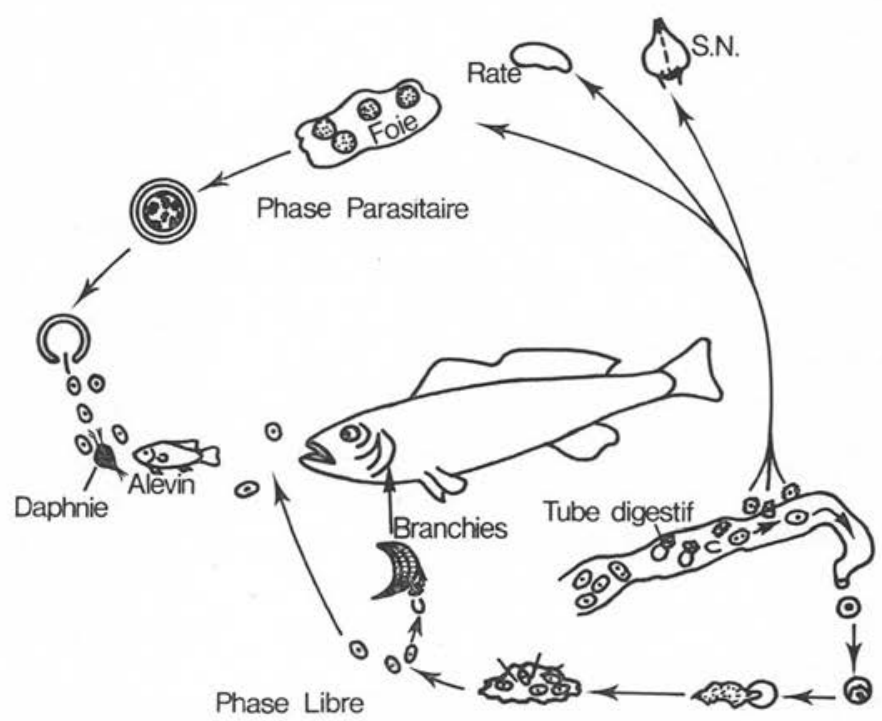

Fig. 6. Cycle évolutif du parasite.

D'autre part, il paraît vraisemblable d'admettre que quelques éléments conidiens binucléés produits par les plasmodes puissent évoluer dans la lumière du tube digestif, comme in vitro, pour donner par caryogamie et par épaississement de leur membrane, des chlamydospores. Ces formes, résistant à l'action des sucs digestifs grâce à leur épaisse paroi $\left(^{*}\right)$ sont rejetées dans le milieu extérieur avec les excréments du poisson.

Trouvant alors, dans les boues sédimentaires, des conditions plus favorables à leur évolution, elles libèrent les éléments plasmodiaux qu'elles contenaient. Une phase végétative libre devient alors possible. Puis les plasmodes "fruotifient », forment des éléments conidiens qui, à leur tour, peuvent être ingérés par des poissons ou par de petits crustacés (Daphnies, par exemple), dont l'infection est ainsi réalisée. On pourrait même admettre la possibilité d'une libération de petits éléments amiboïdes dans l'eau de l'aquarium ou du cours d'eau. Ces éléments infestants pourraient alors

${ }^{*}$ ) Cette résistance a pu être testée in vitro et fera l'objet d'une note ultérieure. 
s'introduire dans de nouveaux hôtes non seulement par voie digestive mais aussi, pensons-nous, par voie trans-muqueuse, au niveau des branchies, ce qui assurerait une dissémination rapide dans le torrent circulatoire.

Quant aux phénomènes de sexualité, il est vraisemblable qu'ils puissent se dérouler aussi pendant cette période de vie végétative libre, dans les boues sédimentaires. Les gamètes issus de sporokystes qui ont assuré leur protection pendant les périodes défavorables procèdent alors, par gamétogamie ou par somatogamie, à la reconstitution du stock diploïde de chromosomes. Les ookystes, à leur tour, libéreront des endospores, diploïdes, multipliant ainsi le nombre de plasmodes dans le milieu extérieur et assurant ainsi la pérennité de l'espèce ( $f$ ig. 7 ).

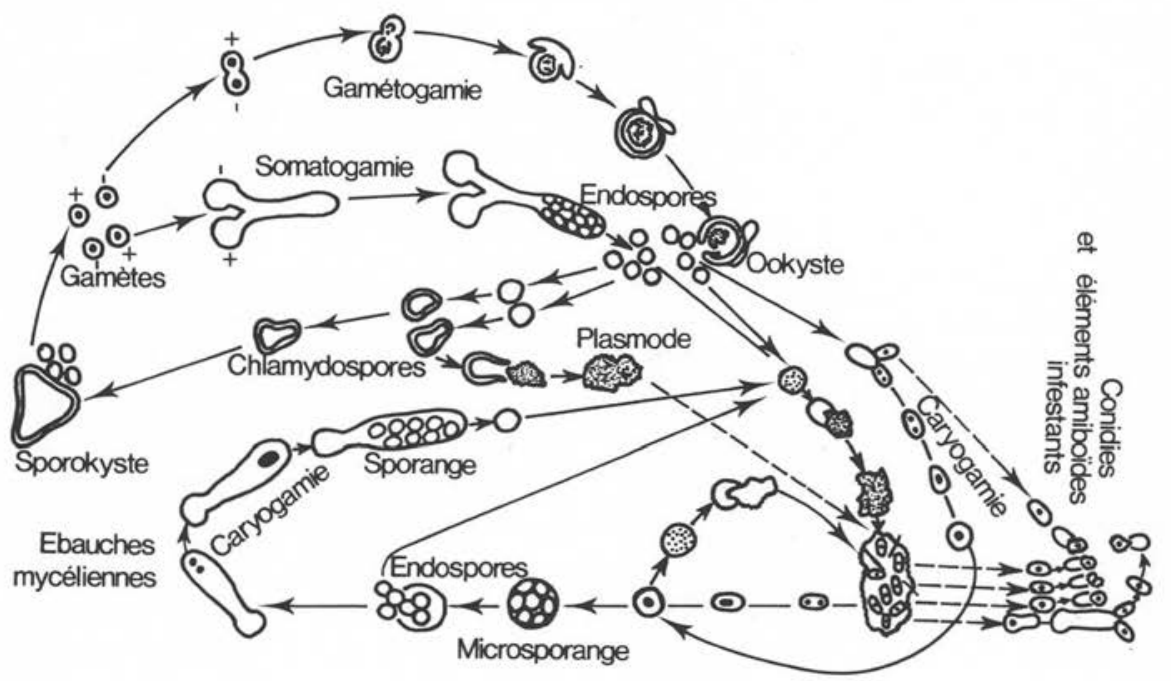

Fig. 7. Phases de la reproduction sexuée et asexuée.

REMERCIEMENTS. - Nous remercions très vivement M. le Professeur J. Biguet, de l'Institut national de la Santé et de la Recherche médicale (Unité de Biologie et d'Immunologie parasitaires et fongiques) qui a bien voulu s'intéresser à nos travaux et nous faire profiter de ses conseils.

Nous remercions aussi MM. les Prs Brygoo et Chabaud, du Muséum national d'Histoire naturelle qui nous ont également encouragé à poursuivre ces recherches.

\section{Bibliographie}

1. Daniel G. E. : Studies on Ichthyophonus hoferi, a parasite of the herring. Am. J. Hyg., 1933, 17, 269-271.

2. Euzeby J.: Cours de Mycologie médicale comparée, 1 vol., 330 p. Vigot, édit., Paris, 1969.

3. Fish F. F.: A fungus disease in fishes in the Gulf of Maine. Parasitology, 1934, 26, 1-16.

4. Johnson T.W. Jr, Sparrow F.K Jr: Fungi in Oceans and Estuaries, (p. 557 et seq.), Hafner Publishing Co, New York, 1961. 